\title{
Relações de gênero e de desempenho físico e motor de alunos submetidos aos testes do eurofit
}

Tânia Maria da Silva Bronsato ${ }^{1}$ e Elaine Romero ${ }^{2}$

\section{Resumo}

Gênero é uma construção social que conduz meninos e meninas a manifestarem diferentes comportamentos na sociedade. Esses comportamentos trazem repercussões no desempenho motor. Assim, o objetivo deste estudo foi verificar se existe diferença no desempenho físico e motor de meninos e meninas, através do Eurofit, averiguando ao mesmo tempo, se existem laços teóricos que expliquem a interferência da cultura nesses desempenhos. Foi realizado um censo com os 118 estudantes de ambos os sexos da $5^{\mathrm{a}} \mathrm{e}$ $6^{a}$ séries do Colégio de Aplicação Dr.Paulo Gissoni. As características do desempenho motor foram obtidas através da bateria de teste Eurofit. A análise estatística percentual apontou que os meninos apresentaram melhores resultados na maioria dos testes.

UNITERMOS: Relações de Gênero; Desempenho Físico e Motor; Atividade Física, Motricidade Humana.

\section{Abstract \\ MOTOR AND PHYSICAL PERFORMANCE IN THE EUROFIT TESTS BY MALE AND FEMALE ELEMENTARY SCHOOL STUDENTS ACCORDING TO GENDER.}

The gender is a social construction which conducis boys and girls to manifest different behavior in the society. These patterns behavior bring repercussions in the motor performance. Thus, the aim of this study was to verify if there is difference in the physical and motor performance of boys and girls through the Eurofit, verifing at the same time if there are theoric ties which explain the interference in these performances. A census with 118 students of both sexes from the $5^{\text {th }}$ and $6^{\text {th }}$ grades of Colégio de Aplicação Dr. Paulo Gissoni was carried out. The characteristics of the motor performance was obtained through the batttery of Eurofit Test. The percentile statistic anlyses showed that the boys got letter results in the most tests.

KEY WORDS: Gender Relations - Motor and Physical Performance - Physical Activity - Human Motricity

\section{Introdução}

A dicotomia masculino/feminino vem suscitando, através da história humana, a discussão sobre os papéis que competem a homens e mulheres na visão sociocultural de todo agrupamento humano organizado. A questão do gênero, presente na tipificação dos sexos, deve ser analisada em relação aos padrões de comportamento, vigentes em cada agrupamento, conforme os valores sociais, políticos, culturais e religiosos, estruturados, ao longo dos séculos.

Em geral, a compreensão do comportamento dos alunos, e em relação às atividades físicas, em particular, não pode ser dissociada do estudo dos estereótipos de gênero que atribuem determinados significados sociais a essas atividades, influenciando as preferências dos alunos e a qualidade das experiências físicas e motoras de meninos e meninas. 
As diferenças bio-psíquicas, com vista à conquista de melhores resultados individuais, sem distinção de sexo, são importantes, também para o processo de melhoria de um padrão de rendimento mais elevado do grupo. Sobre esta relação, em que diferenças biológicas e valores culturais e religiosos determinam o comportamento apropriado ao papel do sexo, persiste o princípio O que se percebe é de que há padrões que a sociedade de preestabeleci-dos ao hoje, ainda é desempenho de hoje, ainda $e_{\text {atividades apropriadas a }}$ organizada em cada sexo, função do homem e especificamente não da mulher. (PIKUNAS, 1987).

Na prática, há uma Sabe-se que da discussão, diversificação de $\begin{aligned} & \text { Sabe-se que da discussão, } \\ & \text { com base na questão do }\end{aligned}$ tratamentos para gênero, à adequação da homens e mulheres, proposta educativa, não perpetuando-se os se pode abandonar modelos, sexualmente preceitos firmados no tipificados pela núcleo social mais família e pela restrito a meninos e sociedade meninas, que é o núcleo parte valores hereditários,

culturais, que moldam a personalidade de cada indivíduo desde o seu nascimento. Para melhores atribuições acerca de questões que envolvem estudos sobre homens e mulheres, determinação biológica e a construção social, emprega-se neste trabalho o termo gênero. Para SCOTT (1990), durante séculos, o termo gênero foi, utilizado de x forma figurada, servindo para distinguir os traços sexuais entre homens e mulheres.

$\mathrm{Na}$ atualidade, o termo "gênero" vem sendo empregado em referência à relação entre os sexos na organização social (SCOTT, 1990). Na visão da mesma autora, a palavra indica uma rejeição ao determinismo biológico, implícito no uso de termos como "sexo ou diferença sexual". Além dis- so, o "gênero" tornou-se uma palavra particularmente útil, pois ele oferece um meio de distinguir a prática sexual, dos papéis sexuais consignados às mulheres e aos homens.

Através desta visão, as diferentes culturas esperam que homens e mulheres tenham papéis e comportamentos distintos, na sociedade (ROMERO, 1994). Assim, baseado num processo socializador, a divisão social dos papéis sexuais inicia-se a partir do próprio nascimento, e se perpetua, ao longo de suas vidas, através das expectativas dos pais e familiares, que buscam tratamento diferenciado, por sexo, e da sociedade que os acolhe.

O que se percebe é que a sociedade de hoje, ainda é organizada em função do homem e não da mulher. Na prática, há uma diversificação de tratamentos para homens e mulheres, perpetuandole os modelos, sexualmente tipificados pela família e pela sociedade. Este cenário é percebido em nosso cotidiano, pois meninos e meninas, em seu convívio familiar, são tratados de forma diferenciada.

Desde que nascem, as crianças passam a ser moldadas em seu papel sexual, claramente definido nas expectativas e percepções de seus pais: a própria cor da roupa do bebê (azul para meninos, rosa para meninas); os tipos de brincadeiras: os meninos brincam na rua, de futebol, bola de gude, pipa, carrinho de rolemã, bicicleta etc; as meninas brincam com bonecas e miniaturas, estimuladas para agir "educadamente" e com delicadezas. Espera-se que os meninos sejam agressivos e atléticos, e as meninas que sejam bonitas, doces, frágeis e delicadas.

Na visão de DAOLIO (1995), estes hábitos corporais masculinos e femininos vão, ao longo do tempo, e dependendo da sociedade, tornando um sexo mais hábil do que o outro, em termos motores. Segundo a Educação Física tradicional é o corpo biológico do indivíduo que tem marcado seu "grupo cultural", separando portanto, homens e mulheres. No entendimento 
de CARDOSO (1994) a Educação Física, em sua especificidade - (o movimento) - pode estimular ações que venham contribuir com posturas adequadas a cada indivíduo, sem distinção de sexo, permitindo que sua potencialidade motora o habilite a fugir de estereótipos, instalados social e culturalmente. Assim sendo, a Educação Física, como elemento do sistema geral de Educação, utiliza como meios principais os exercícios físicos com a finalidade de contribuir para a adaptação biológica e social do indivíduo ao meio, e, ainda para afirmação de sua personalidade (BARROS, 1986).

No entanto, a socialização entre os sexos, tal como ainda ocorre em aulas de Educação Física, gera a oposição, e não interação de ações. A prática conjunta de meninos e meninas pode ser uma das difíceis tarefas da Educação Física, dada à discriminação de papéis masculino e feminino, instalados socialmente.

Com isso, os valores estabelecidos e mantidos pelos agentes socializadores, na forma de modelos reais e simbólicos e os papéis sexuais masculinos e femininos, que permeiam na sociedade brasileira, fazem com que a mulher, com relação a atitudes e valores, seja submissa e discriminada (ROMERO, 1995). Isso, provavelmente, deve-se ao fato histórico/cultural que dificultou, ao longo dos séculos, o acesso das mulheres às diversas atividades sociais. Cada tipo de sociedade tem sua visão diferenciada de entender o "corpo" e o comportamento social das mulheres, principalmente nos países da América Latina.

O presente estudo buscou também rever antigos conceitos sobre a adequação de atividades, em Educação Física segundo o sexo, na tentativa de apontar a necessidade de serem substituídos por uma ação pedagógica, conveniente e favorável ao desempenho físico e motor de ambos os sexos.

Nesse entendimento, procuramos verificar se existe diferença no desempenho físico e motor de meninos e meninas da $5^{\text {a }}$ e $6^{\text {a }}$ séries do ensino fundamental, quando submetidos a uma bateria de teste Eurofit, averiguando, ao mesmo tempo, a existência de laços teóricos que expliquem a interferência da cultura nesses desempenhos.

\section{Gênero e motricidade humana}

Hoje, a questão de gênero assume o sentido de representar a construção social e histórica dos sexos, enfatizando-se o caráter social e relacionai desta construção. Reforça-se, assim que a diferença biológica, entre os sexos e o meio social, define os padrões de comportamento, adequando-se e interrelacionando-se estes padrões, sem supremacia de um sexo sobre o outro. Assim, o comportamento sexual humano se expressa no contexto de uma cultura e dos modos padronizados de se viver, que caracterizam a vida social.

É nesse sentido que BIAGGIO (1985) esclarece que os padrões de desenvolvimento das preferências de papéis masculinos e os femininos são diferentes, segundo a classe social.

Para WEBER (1997), uma vez estabelecidos os papéis sociais a serem desempenhados pelos sexos masculino e feminino - as relações sociais de gênero - parecem ser mais fáceis adequarem-se a eles do que forçar mudanças em oposição aos papéis sociais determinados.

Nesse contexto, as diferenças significativas, relacionadas ao papéis masculino e feminino, são motivo de análise, como, por exemplo, para ROMERO (1995), que ressalta ser na família que esse processo se inicia, pois compete aos pais a decisão sobre quais atividades são adequadas, ou não, a seus filhos, segundo o sexo. Ou seja, as crianças assimilam, desde o nascimento, o que é próprio ou impróprio ao seu sexo, segundo as normas culturais, levando essa aprendizagem para o restante da vida.

Nesse sentido, os meninos e as meninas desde muito cedo possuem suas vidas delineadas a par- 
tir das experiências que originarão seus hábitos, condicionamentos e identificação. $\mathrm{O}$ constructo "identificação" tem papel central em várias teorias de desenvolvimento da personalidade (BIAGGIO, 1985). No processo de identificação que acontece, ao longo da infância e mais na adolescência, por seleção de atitudes dos modelos masculino e feminino, presentes na figura do pai e da mãe, é que a criança firma o seu papel na sociedade.

\section{Para}

BELOTTI

$O$ estudo $(1985$, p.8) "o objetivo da Motricidade da identificação de Humana, revela as uma criança com o diversas manifestações sexo para o qual a dedo comportamento com bastante rapidez e motor do indivíduo não existem elementos sempre na para deduzir que este dependência da complexo fenômeno intencionalidade de tenha raízes biológicas". cada Ser. Desse modo, compreender e avaliar desenvolve-se cada um papel sexual não indivíduo, quer na como uma característica condição fisica, quer de personalidade, na biológica, em sua mas como expressão totalidade de uma cultura.

Compreende-se assim que as diferenças estabelecidas culturalmente tornam-se fáceis ao reconhecimento do que pertence ao indivíduo, em uma ou outra cultura: este é um menino! ou esta é uma menina! Contudo, na concepção do Ser, BRUHNS (1995) diz que o corpo humano é uma parte muito especial do universo, pois nele "habita" o próprio indivíduo que percebe o mundo.

Transcendendo a diversidade anatômica e fisiológica, a percepção do corpo pode nos conduzir a uma diferenciação de gênero (BRHUNS, 1995). Ainda, segundo Merleau-Ponty (apud CUNHA, 1994), o homem, através da cultura, recebe a experiência do passado e aprende a preparar o futuro. Realiza-se e compreende-se na e desde a experiência.

Em se tratando da razão principal desde tudo, o desempenho motor de meninos e meninas, entende-se que a motricidade deve ser analisada como processo adaptativo, evolutivo e criativo de um ser prático, carente dos outros, do mundo e da transcendência (CUNHA, 1994). A motricidade é a condição que permite a cada Ser Humano, em que as condições próprias, física, mental, emocional e espiritual, possam interagir, tornando-o um Ser Humano global e Uno.

O estudo da Motricidade Humana, revela as diversas manifestações do comportamento motor do indivíduo sempre na dependência da intencionalidade de cada Ser. Desse modo, desenvolve-se cada indivíduo, quer na condição física, quer na biológica, em sua totalidade.

Entende-se, assim, que a aplicação do conceito de gênero, quanto à motricidade do homem e da mulher, se firma na razão direta da necessidade de se transcender as limitações do indivíduo, como Ser único, e em interação com o meio a que pertence. Meninos e meninas têm desenvolvimento motor diferenciado como seres únicos e que podem interagir, na busca de seus padrões ideais de comportamento e de desempenho, em atividade de Educação Física.

\section{O desempenho motor e a necessidade de um processo de aprendizagem}

No mundo contemporâneo, entretanto, a dimensão social assume um importante papel na explicação do comportamento humano. Orlick (apud BRANDÃO e FIGUEIRA JÚNIOR, 1996) definiu o comportamento humano como uma função de sua natureza biológica e da aprendizagem, que é resultante da interação com o meio ambiente, particularmente com o ambiente social. 
Por sua vez, o desempenho motor de crianças e adolescentes constitui-se numa preocupação permanente entre os especialistas da área de saúde (GUEDES e BARBANTI, 1995). Este interesse se justifica na medida em que a atividade física pode desempenhar importante papel na prevenção, conservação e melhoria da capacidade funcional, e por conseguinte, na saúde dos jovens (Power e Paffenbarger, apud BARBANTI, 1995).

Sabendo-se que o nível de desempenho motor ou performance física depende da quantidade e qualidade das vivências motoras, meninos e meninas deverão ser encorajados por agentes de socialização a empenharem-se em atividades de toda a natureza. Assim, faz-se necessário um processo de ensino-aprendizagem em Educação Física, que capacite os meninos e meninas a perceberem suas possibilidades de aprendizagem e desempenho corporal e, com autonomia, exercê-las de maneira adequada, social e culturalmente significativa.

$\mathrm{O}$ equilíbrio biológico-funcional do organismo ravorece o desenvolvimento normal de uma pessoa para o ato de aprender. No entanto, somente estímulos significativos (que atendam suas reais necessidades) darão a este indivíduo motivos para que o momento da aprendizagem resulte em benefício próprio a este determinado indivíduo.

Para que a aprendizagem ocorra, é preciso na ação educativa de pais e na ação pedagógica dos professores que a criança não seja tolhida em sua exploração, e que tenha um ambiente propício para o desenvolvimento de suas potencialidades, independentemente de seu sexo biológicamente determinado ou do gênero socialmente construído. A socialização em um papel social específico envolve a aquisição de habilidades (motoras e sociais), valores, conhecimentos, atitudes, normas e disposições que podem ser aprendidas em uma ou mais instituições sociais (PELLEGRINI, GREENDORFER e BLINDE, 1987).

Desta forma, a compreensão do comportamento das crianças, em geral, e em relação a aprendiza- gem das atividades motoras e esportivas, não se pode dissociar do estudo dos estereótipos de gênero que atribuem determinados significados sociais a essas atividades, influenciando as preferências das crianças e a qualidades das experiências motoras de meninos e de meninas.

\section{Atividade física ou desempenho físico e diferenças individuais entre meninos e meninas}

Segundo CORBIN (1987), a atividade física é considerada como o processo do qual resultará o estado de aptidão física do indivíduo.

De acordo com BOHME (1993), a aptidão física é demonstrada pela capacidade do indivíduo apresentar um desempenho físico adequado em suas atividades físicas diárias. Na visão da mesma autora (1994), a aptidão física, vista como produto resultante da atividade física, deve ser

desenvolvida durante to-
das as fases da vida do ser humano, visando mito masculino e proporcionar-lhe umfeminino é desempenho físicoindispensável adequado nas suasdenunciar que a atividades diárias. Para Educação Física entender as diferenças mesmo nos dias de individuais de meninos e hoje, mantém os desempenho físico epapéis sexuais motor é de grandedistintos e importância, pois estadeterminados, fornece informação para caracterizando os co o processo ensi-no- mportam en tos aprendizagem.

masculino e feminino,
a serviço de uma
ideologia sexista

A faixa etária em discussão atinge crianças entre 10 e 14 anos de idade que estão em constantes mudanças físicas e psicológicas, não podendo, 
seu desenvolvimento, estar isolado do contexto social, cultural e educacional no qual sua motricidade é exercida. Assim a atividade física, pode colaborar de forma significativa na educação de meninos e meninas, possibilitando um crescimento harmônico do organismo, enfocando o desempenho físico e motor nas diversas provas motoras.

Para DANTAS (1987), o desporto é um importante meio de integração social e para Embora existam POMPEU (1995), a pequenas diferenças idade correta para o início nas trajetórias dos da especialização do treisegmentos perna, coxa ponto importante é um e tronco entre os futura vida atlética. sujeitos, uma menor Wanne e Valimak (apud variabilidade for POMPEU, 1995), dizem detectada no grupo de que o treinamento de adultos, sendo que estes apresentaram um padrão mais consistente endurece não influência o crescimento de crianças do sexo masculino e feminino.

Quanto à diferença de força entre meninos e meninas pré-púberes, esta parece ser apenas um fenômeno social conforme afirma POMPEU (1995). Quando as meninas utilizam a força dos braços e ombros para resistirem à gravidade apresentam desempenho similar ao dos meninos.

KNAPP (s/d) reforça essa diferenciação, ressaltando que nos rapazes durante a adolescência, produz-se um rápido crescimento de força e, pelo fato desta representar um importante fator na performance, os indivíduos com desenvolvimento tardio serão novamente desfavorecidos.

ROMERO (1994) afirma que em se tratando do mito masculino e feminino é indispensável denunciar que a Educação Física mesmo nos dias de hoje, mantém os papéis sexuais distintos e determinados, caracterizando os comportamentos masculino e feminino, a serviço de uma ideologia sexista. Dentro deste contexto, MYOTIN (1995) observou que os índices de prática feminina decrescem com a idade durante a adolescência influenciados pelo processo de socialização que desencoraja a prática de esportes pelas meninas à medida que elas amadurecem.

Tendo em vista a revisão de literatura, o objetivo deste estudo é verificar se existe diferença no desempenho físico e motor de meninos e de meninas, através do Eurofit, averiguando, ao mesmo tempo, se existem laços teóricos que expliquem a interferência da cultura nesses desempenhos.

\section{Material e método}

Participaram do estudo todos os estudantes os 118, com idade compreendida entre 10 e 14 anos, freqüentando regularmente a $5^{\mathrm{a}}$ ou $6^{\mathrm{a}}$ séries do Colégio de Aplicação Dr. Paulo Gissoni da Universidade Castelo Branco (RJ), sendo que destes 59 eram meninos e 59 meninas.

Como instrumentos foram empregados: estadiômetro, balança com toeza e estadiômetro, compasso de dobras cutâneas. Foi aplicada uma bateria de testes motores, denominada Eurofit, composta de teste de equilíbrio (flamingo), teste tocando os discos, teste sente e alcance, teste salto em extensão, teste de força de apreensão manual, teste sente e levante, teste de suspensão com o braço flexionado, teste Shuttle Run, teste de resistência cardio-vascular (endurance) - teste de Cooper. Os dados foram registrados em uma ficha de coleta de dados do Eurofit.

Para a coleta dos dados os instrumentos foram levados à escola, onde os grupos foram testados em uma ordem aleatória, em três ambientes (laboratório, quadra e pista de atletismo), em três semanas, sendo que em cada uma delas foi escolhido o dia em que os alunos tinham aula de Educação Física.

Foram tomadas as medidas antropométricas e 
dobras cutâneas no primeiro dia de coleta de dados. Foram seqüenciados na seguinte ordem: altura, peso, dobras cutâneas (composição corporal). Para a mensuração das dobras cutâneas segui-se o protocolo do Eurofit a saber: dobra cutânea triceptal, biceptal, subescapular, suprailíaca e de panturrilha.

No segundo dia de coleta dos dados passou-se à bateria de testes motores, composta por oito itens: a) Teste de equilíbrio - FLB (objetivo: medir o equilíbrio total no corpo); b) Tocando os discos PLT (objetivo: medir a velocidade de membros superiores); c) Sente e alcance - SAP (objetivo: mensurar a flexibilidade de tronco); d) Salto/extensão parado - SBJ (objetivo: medir força explosiva de membros inferiores); e) Força de apreensão manual - HGR (objetivo: mensuração da força estática de mão); f) Sente e levante - SUP

(objetivo: avaliar força de tronco - abdominal); g). Suspensão com o braço flexionado - BAH (objetivo: avaliar força dos membros superiores); h) Shuttle Run - SHR (objetivo: mensurar velo-cidade de corrida - agilidade). Não foi permitido aos alunos fazerem nenhum ensaio preliminar dos testes e nenhum exercício de alongamento ou aquecimento antes do início dos testes motores, exceto o teste de Cooper.

finalmente, no terceiro dia de coleta de dados, foi efetuada a avaliação cardio-respiratória, por meio do consumo máximo de oxigênio, obtido de forma indireta, no teste andar e correr em 12

\begin{tabular}{|c|c|c|c|c|c|c|}
\hline $\begin{array}{l}\text { TABELA } \\
\text { sm funcãe }\end{array}$ & $\begin{array}{l}\text { l: Re: } \\
\text { do se? }\end{array}$ & $\begin{array}{l}\text { uhta } \\
\text { (N }\end{array}$ & em. & do & de & ih \\
\hline Escuilihrio & Fem & butino & $\overline{M a}$ & ulino & & \\
\hline Pontos & Abs. & $\%$ & Abs. & 90 & Abs. & 90 \\
\hline $2 \mid-3$ & 4 & $7 \%$ & 0 & $0 \%$ & 4 & $3 \%$ \\
\hline $3,-4$ & 4 & $7 \%$ & 1 & $2 \%$ & 5 & $4 \%$ \\
\hline $4 \cdot-5$ & 8 & $149 \%$ & 3 & $5 \%$ & 11 & $9 \%$ \\
\hline $5+6$ & 9 & $15 \%$ & 8 & $14 \%$ & 17 & $14 \%$ \\
\hline $61-7$ & 11 & $19 \%$ & 8 & $14 \%$ & 19 & $16 \%$ \\
\hline $7 \mid-8$ & 10 & $17 \%$ & 10 & $17 \%$ & 20 & $17 \%$ \\
\hline $81-9$ & 9 & $15 \%$ & 17 & $29 \%$ & 26 & $229 \%$ \\
\hline $9-10$ & 4 & $7 \%$ & 12 & $20 \%$ & 16 & $14 \%$ \\
\hline Total & 59 & $100 \%$ & 59 & $100 \%$ & 118 & $100 \%$ \\
\hline
\end{tabular}

minutos, segundo a proposta de COOPER (1986). Antes de iniciar a tarefa andar e/ou correr, em cada sessão foi verificada a freqüência cardíaca em repouso (inicial), e realizado um aquecimento, com duração de 5min., conforme orientação de COOPER (1982). Ao término do teste verificou-se a freqüência máxima, para melhor controle fisiológico.

Para o tratamento dos dados foi utilizada a estatística descritiva.

\section{Resultados e discussão}

Considerando-se que foi investigada a totalidade da população, foi utilizada a estatística descritiva no intuito de permitir a compreensão da distribuição do comportamento das variáveis relevantes ao estudo, por meio do resumo dos dados em tabelas e figuras, bem como, no cálculo de medidas estatísticas.

Iniciando-se a análise dos dados do Eurofit, a TABELA 1 permite o estudo pormenorizado dos resultados obtidos no teste de equilíbrio. Como se vê, os sujeitos do sexo feminino alcançaram, em sua maioria (80\%), de 4 a 9 pontos. No que se refere ao grupo masculino, a maior concentração distribui-se na faixa de 5 a 10 pontos, o que corresponde, aproximadamente a $94 \%$ dos sujeitos com valores mais altos, o que aponta diferença favorável aos meninos.

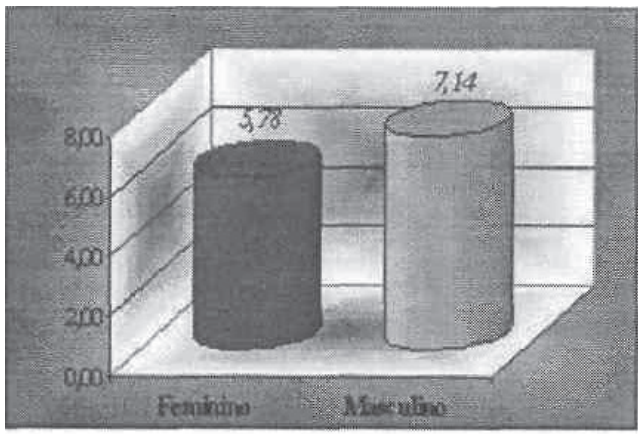

FIGURA 1: Media dos valor es obtidos no teste de equilibrio em funç̃o do sexo. 


\begin{tabular}{|c|c|c|c|c|c|c|}
\hline $\begin{array}{l}\text { TABEL } \\
\text { liscos a }\end{array}$ & $\begin{array}{l}\text { Re } \\
\text { uncá }\end{array}$ & ed & of & ste & & \\
\hline Tocando & Fem & mino & Mas & ulino & & tal \\
\hline $\begin{array}{l}\text { os Discos } \\
\text { (dec.Seg) }\end{array}$ & Abs. & $\%$ & Abs. & $\%$ & Abs. & $\%$ \\
\hline $100 \mid-110$ & 0 & $0 \%$ & 2 & $3 \%$ & 2 & $2 \%$ \\
\hline $110 j-120$ & 10 & $17 \%$ & 9 & $15 \%$ & 19 & $16 \%$ \\
\hline $120 \%$ & 20 & $34 \%$ & 20 & $34 \%$ & 40 & $34 \%$ \\
\hline $130 \mid-140$ & 15 & $25 \%$ & 15 & 259 & 30 & $25 \%$ \\
\hline $140:-130$ & 7 & $12 \%$ & 6 & $10 \%$ & 13 & $11 \%$ \\
\hline $150:-160$ & 3 & $5 \%$ & 3 & $5 \%$ & 6 & $5 \%$ \\
\hline $160 \mid-170$ & 2 & $3 \%$ & 3 & $5 \%$ & 5 & $4 \%$ \\
\hline $1701-180$ & 2 & $3 \%$ & 1 & $2 \%$ & 3 & $3 \%$ \\
\hline Total & 59 & $100 \%$ & 59 & $100 \% 8$ & 118 & $100 \%$ \\
\hline
\end{tabular}

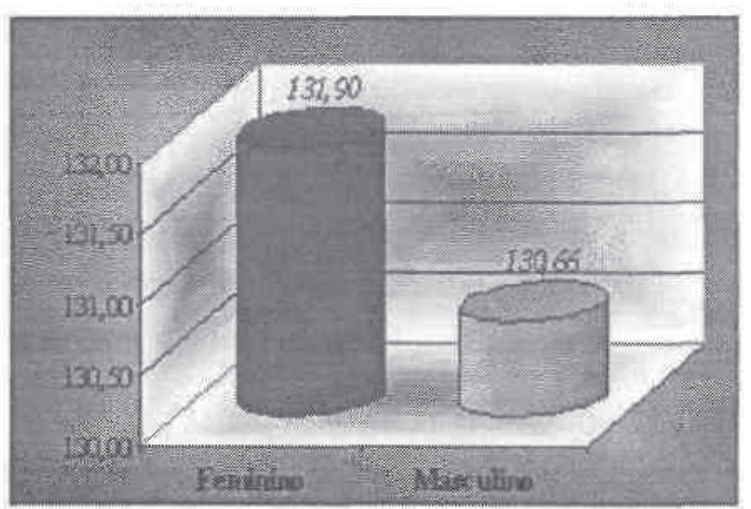

FIGURA 2: Média dos valores do teste tocendo os discos em funçto do sexo

Na segunda prova estudada, "tocando os discos", a TABELA 2 ilustra o desempenho motor de ambos os sexos, no qual pode-se verificar um desempenho médio um pouco superior no grupo masculino, embora as duas distribuições estejam com comportamento bastante semelhante.

Neste teste, o objetivo era velocidade de membros superiores, num menor tempo, sendo este um fator primordial. Para este objetivo, os dados apontam que os meninos, alcançaram uma média percentual acima daquela obtida pelas meninas, mas sem indicação de diferença marcante entre os grupos.

Analisando a FIGURA 2, vê-se que a média do grupo feminino se apresenta com um quantitativo de 131,90 décimos de segundo, enquanto para o masculino de 130,66 décimos de segundo. Reportando-nos à literatura, BARBANTI (1996) afirma que

\begin{tabular}{|c|c|c|c|c|c|c|}
\hline \multicolumn{7}{|c|}{$\begin{array}{l}\text { TABELA 3: Resultado em \% do teste sente } \\
\text { alcarce em fumcáo do sewo (N=118) }\end{array}$} \\
\hline \multirow{2}{*}{$\begin{array}{l}\text { Sente e } \\
\text { Alcance } \\
\text { (cm) }\end{array}$} & \multicolumn{2}{|c|}{ Feminive } & \multicolumn{2}{|c|}{ Masculino } & \multicolumn{2}{|c|}{ Total } \\
\hline & Abs. & $\%$ & Abs. & $\%$ & Abs. & $\%$ \\
\hline $119 \mid-159$ & 3 & $5 \%$ & 5 & $8 \%$ & 8 & $7 \%$ \\
\hline $1591-199$ & 2 & $3 \%$ & 6 & $10 \%$ & 8 & $7 \%$ \\
\hline & 5 & $8 \%$ & 14 & $24 \%$ & 19 & $10 \%$ \\
\hline $239-279$ & 19 & $32 \%$ & 12 & $20 \%$ & 31. & $20 ?$ \\
\hline $279 \mid-319$ & 13 & $22 \%$ & 13 & $22^{\circ}$ & 26 & $22 \%$ \\
\hline $319 \mid-359$ & 11 & $19 \%$ & 9 & $15 \%$ & 20 & $17 \%$ \\
\hline $359 \mid-399$ & 5 & $8 \%$ & 0 & $0 \%$ & $1=1$ & $4 \%$ \\
\hline $399 / 439$ & 1 & $2 \%$ & 0 & $Q \%$ & , & $1 \%$ \\
\hline Toial & 59 & $100 \%$ & 59 & $100 \%$ & 118 & $100^{\circ}$ \\
\hline
\end{tabular}

"as valencias como flexibilidade, agilidade $\epsilon$ podem ser trabalhadas de maneiras iguais 1 os sexos". Algumas das atividades praticada meninos em seu dia a dia, com envolvime componentes físicos, poderia ter contribuíc que eles apresentassem um desempenh centualmente melhor do que as meninas, $\mathrm{c}$ isso não foi encontrado no presente estudo.

Dando seqüência aos resultados, observa TABELA 3 os sujeitos do sexo feminino ram um grau de flexibilidade maior que o $d$ masculino.

Nota-se que neste teste as meninas obtivera: média de flexibilidade no valor de 281,7 os meninos uma média de $251,78 \mathrm{~cm}$ com tra a FIGURA 3. AZEVEDO (1997), cc porte em Mead (1971) e em Mota (1985),

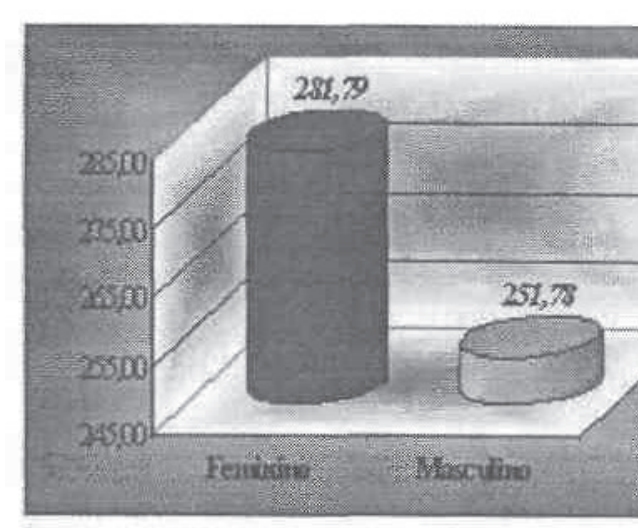

FIGURA 3: Média dos val ares obtidos n teste sente e alcance em funçăo do sexo 


\begin{tabular}{|c|c|c|c|c|c|c|}
\hline $\begin{array}{l}\text { TABELA } \\
\text { extensão s }\end{array}$ & $\begin{array}{l}4: 5 \\
\text { corra }\end{array}$ & Has & 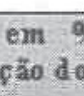 & do & 6 & 0 \\
\hline $\begin{array}{l}\text { Salto em } \\
\text { Extersấo } \\
\text { (cm) }\end{array}$ & Fem & inine & Masx & ulino & & tal \\
\hline & Abs: & 85 & Abs. & $\%$ & Abs. & 96 \\
\hline $108 \mid-123$ & 20 & $34 \%$ & 3 & $5 \%$ & 23 & $19 \%$ \\
\hline $123 \mid-138$ & 22 & $37 \%$ & 14 & $24 \%$ & 36 & $31 \%$ \\
\hline $138 \mid-153$ & 5 & $8 \%$ & 22 & $37 \%$ & 27 & $23 \%$ \\
\hline$|53|-168$ & 7 & $12 \%$ & 9 & $15 \%$ & 16 & $14 \%$ \\
\hline $1681-183$ & 3 & $5 \%$ & 3 & $12 \%$ & 10 & $8 \%$ \\
\hline $183 \mid-198$ & 2 & $3 \%$ & 2 & $3 \%$ & 4 & $3 \%$ \\
\hline $1981-213$ & 0 & $0 \%$ & 1 & $2 \%$ & 1 & $18 \%$ \\
\hline $2131-21$ & 0 & $0 \%$ & 1 & $2 \%$ & 1 & $1 \%$ \\
\hline Total & 59 & $100 \%$ & 59 & $100 \%$ & 118 & $100 \%$ \\
\hline
\end{tabular}

ca que os papéis sexuais são, antes de tudo, aquisições culturais e não, unicamente, resultante de fatores orgânicos. Na continuidade de sua linha de pensamento e servindo-se de Oglerby (1982), ressalta que a "flexibilidade de maneira geral, é considerada socialmente como um atributo do sexo feminino". AZEVEDO (op.cit), aponta que, de maneira geral, as mulheres são mais flexíveis em todas as faixas de idade.

A TABELA 4 apresenta as informações dos dados obtidos no salto em extensão/sem corrida, detalhando o desempenho masculino e feminiao. Numa visão geral, no que diz respeito à valencia força de membros inferiores, tem-se que $71 \%$ dos sujeitos do sexo feminino apresentou medidas em torno de 108 a $138 \mathrm{~cm}$ no salto em extensão/sem corrida. Os sujeitos do sexo masculino, que correspondem a um percentual de $88 \%$ de representatividade exibem medidas de 123 a $183 \mathrm{~cm}$. Sendo assim, os dados indicam melhor desempenho masculino, em relação ao reminino.

Na visão de Wilmore (apud AZEVEDO, op. cit., p.117), a mulher, da mesma forma que o homem, apresenta treinabilidade para sobrecarga (força muscular). A partir destas informações pode-se deduzir que atividades que envolvem rorça, são independentes do sexo. Esse fato parece indicar que a influência do meio social é bas-

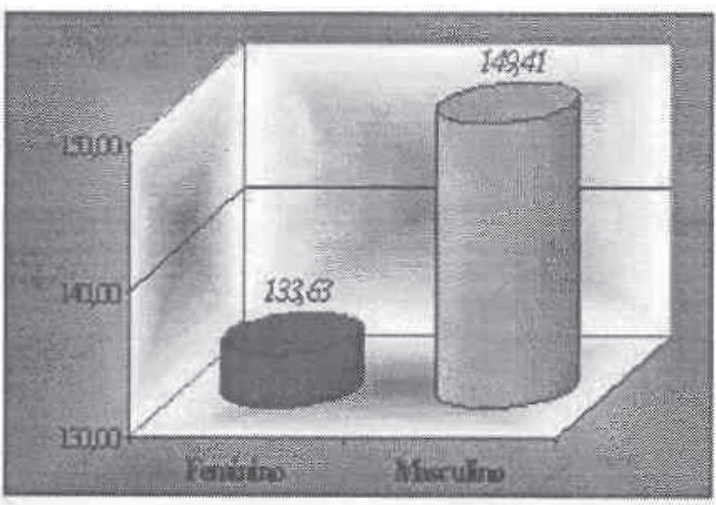

FIGURA 4: Média dos valores obtidos no teste salto em extenão s/corricia em função cio sexo

tante relevante no desenvolvimento da força muscular, e que esta valencia pode ser trabalhada de maneira igual nos dois sexos. Porém, o sexo masculino obteve melhor resultado. Diante dos dados obtidos, pode-se inferir que as meninas não tiveram semelhante resultado porque muitas vezes ao longo de suas vidas, obtiveram seu desempenho motor comprometido, em comparação com os meninos, em razão do mito da feminilidade. Este desaconselhava às mulheres atividades esportivas que envolvessem trabalho de força muscular.

Percebe-se na FIGURA 4, que no teste salto extensão/sem corrida, a média dos sujeitos do sexo feminino se mostra com o valor de $133,63 \mathrm{~cm}$ e $149,41 \mathrm{~cm}$ para o masculino.

Prosseguindo a análise estudou-se os resultados do teste de apreensão manual e para tanto, elaborou-se a TABELA 5. Foi possível verificar que em termos de freqüências absolutas e relativas (\%), 64\% dos meninos tiveram resultados concentrados em torno de $8 \mathrm{~kg}$ a $20 \mathrm{~kg}$. Os outros $34 \%$ dos meninos tiveram resultados dispersados acima dos $20 \mathrm{~kg}$ e abaixo dos $32 \mathrm{~kg}$. Esses percentuais, quando somados, perfazem $98 \%$ do grupamento masculino. Em relação às meninas, verificou-se que $90 \%$ teve resultados que na mesma tabela, ficaram concentrados entre $8 \mathrm{~kg}$ e $20 \mathrm{~kg}$. Os $10 \%$ restantes ficaram dispersos entre valores acima de $20 \mathrm{~kg}$ e abaixo dos $26 \%$. 


\begin{tabular}{|c|c|c|c|c|c|c|}
\hline $\begin{array}{l}\text { TABELA } \\
\text { manual ex }\end{array}$ & $\begin{array}{l}\text { 5. Re: } \\
\text { i fusça }\end{array}$ & $\begin{array}{l}\text { suliad } \\
\text { io de s }\end{array}$ & $\begin{array}{l}\text { em of } \\
\cos (\mathrm{N}=1\end{array}$ & $\begin{array}{l}\text { do } \\
\text { 18) }\end{array}$ & de & ee \\
\hline Ap reensäo & Fem & iniro & Masc & uline & & tol \\
\hline $\begin{array}{l}\text { Manual } \\
\text { (kgf) }\end{array}$ & Abs. & 96 & Abs. & 96 & Abs. & $\%$ \\
\hline $8 \mid-11$ & 7 & $12 \%$ & 4 & $7 \%$ & 11 & $9 \%$ \\
\hline 111.14 & 20 & $34 \%$ & 13 & $22 \%$ & 33 & $28 \%$ \\
\hline$|4|-17$ & 14 & $34 \%$ & 15 & $25 \%$ & 29 & $25 \%$ \\
\hline $17 \mid-20$ & 12 & $20 \%$ & 10 & $17 \%$ & 22 & $19 \%$ \\
\hline $201-23$ & 5 & $8 \%$ & 4 & $7 \%$ & 9 & $8 \%$ \\
\hline $231-26$ & 1 & $2 \%$ & 3 & $12 \%$ & 8 & $7 \%$ \\
\hline $26 \mid 29$ & 0 & $0 \%$ & 1 & $2 \%$ & 1 & $1 \%$ \\
\hline $291-32$ & 0 & $0 \%$ & 5 & $8 \%$ & 5 & $4 \%$ \\
\hline Total & 59 & $100 \%$ & 59 & $100 \%$ & 118 & $100 \%$ \\
\hline
\end{tabular}

Para melhor ilustrar, elaborou-se a FIGURA 5, na qual se bem nota que a média das meninas ficou em torno de $14,56 \mathrm{~kg}$, enquanto que, para os meninos ficou em torno de $17,47 \mathrm{~kg}$. Segundo Mathews,Campos e Wilmore (Apud AZEVEDO, 1997, p.1 17), os homens são, em termos absolutos, mais fortes, cerca de $30 \%$ a $40 \%$ do que as mulheres. Os achados do presente estudo se assemelham aos dos apontados por AZEVEDO (op.cit)

Por outro lado POLLOCK eWILMORE (1993, p.209), discorrendo sobre treinamento para força em mulheres, levantam questões relacionados ao que comumente se considera sexo frágil e estereotipado. Na prática diária observa-se que meninos e meninas praticam de alguma forma, exercícios ou atividades que desenvolvem a força muscular, mas, por influência do meio social, as

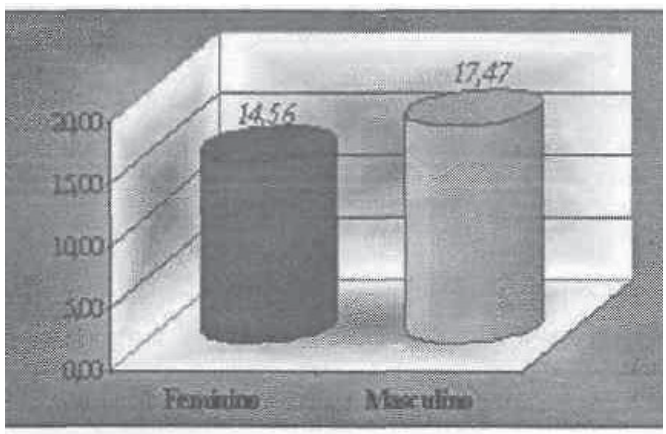

FIGURA S: Média dos valores obtidos no teste de apreensäo manual em funç̧̄o do sexo

meninas são levadas, desde cedo, a dese pouco a força muscular.

Pelos dados analisados nesta prova, cons 1 que os índices atingidos em relação à for tica de mão foram favoráveis ao sexo mas

Analisou-se, a seguir, os resultados obti teste sente e levante, que avaliou a valenc abdominal. Os dados na TABELA 6 con formações sucintas sobre o desempenho bos os sexos. Percebe-se que os percentuc dos denotam um desempenho motor fa para o sexo masculino. Para melhor comp a FIGURA 6 espelha que a média das $m$ ficou em torno de 15,05 repetições abdon enquanto para os meninos a média foi de

Araújo (apud AZEVEDO, 1997, p.l 17) que "a força muscular por área de seção trc

\begin{tabular}{|c|c|c|c|c|c|c|}
\hline \multicolumn{7}{|c|}{$\begin{array}{l}\text { Tabela 6: Resultado em \% do teste sente e levante em } \\
\text { funcäo do sexo }(\mathrm{N}=118)\end{array}$} \\
\hline Sente $\mathrm{e}$ & \multicolumn{2}{|c|}{ Feminino } & \multicolumn{2}{|c|}{ Masculino } & \multicolumn{2}{|c|}{ Total } \\
\hline $\begin{array}{l}\text { Levante } \\
\text { (Repet- } \\
\text { 30s) }\end{array}$ & Abs. & $\%$ & Abs. & $\%$ & Abs. & $\%$ \\
\hline $81-11$ & 4 & $7 \%$ & 2 & $3 \%$ & $\frac{6}{6}$ & $5 \%$ \\
\hline $11 \mid-14$ & 17 & $29 \%$ & 4 & $7 \%$ & 21 & $18 \%$ \\
\hline $14 \mid-17$ & 20 & $34 \%$ & 15 & $25 \%$ & 35 & $30 \%$ \\
\hline $17-20$ & 12 & $30 \%$ & 17 & $29 \%$ & 29 & $25 \%$ \\
\hline $20 \mid-23$ & 6 & $10 \%$ & 10 & $17 \%$ & 16 & $14 \%$ \\
\hline $23 \mid-26$ & 0 & $0 \%$ & 5 & $8 \%$ & 5 & $4 \%$ \\
\hline $261-29$ & 0 & $0 \%$ & 4 & $7 \%$ & 4 & $3 \%$ \\
\hline $291-32$ & 0 & $0 \%$ & 2 & $3 \%$ & 2 & $2 \%$ \\
\hline Total & 59 & $100 \%$ & 59 & $100 \%$ & 118 & $100 \%$ \\
\hline
\end{tabular}

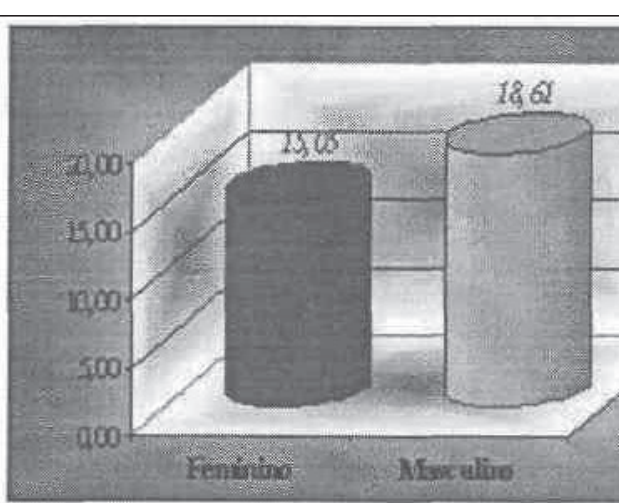

FIGURA 6: Média dos valares obtidos no 1 sente e levante em função do sex 0. 
sal do músculo de igual tamanho, igual tipo de fibras, tende a ser idêntica entre os sexos". A pouca literatura encontrada sobre o assunto não oferece condições para inferir comentários sobre os resultados encontrados.

O próximo teste estudado objetivou avaliação de força dos membros superiores, suspensão de braços. Para a análise construi-se a TABELA 7, na qual se pode observar os resultados obtidos para ambos os sexos. Vê-se então, que $93 \%$ do sexo ferninino apresentou resultados entre os valores compreendidos de 0,1 a 1,1 (déc. de seg.), ao passo que $80 \%$ do masculino logrou resultados que oscilaram neste mesmo intervalo. Acima deste ficaram os $20 \%$ restantes do grupo masculino .

Na FIGURA 7, pode-se averiguar que no confronto das médias entre os sexos, neste teste, os meninos evidenciaram melhor resultado. Estes dados iparecem indicar que "a influência do meio social é bastante relevante no desenvolvimento da força muscular, posto que, desde criança, as mulheres são pouco solicitadas para atividades que envolvam força do braço" (AZEVEDO, 1997).

Para análise do desempenho do sexo masculino e reminino no teste de agilidade, elaborou-se a

TABELA 8 que permite estudo detalhado, em termos percentuais dos resultados obtidos.

Conforme os dado coletados, verificou-se que $87 \%$ dos meninos tiveram maior concentração

\begin{tabular}{|c|c|c|c|c|c|c|}
\hline \multicolumn{7}{|c|}{$\begin{array}{l}\text { TABELA 7: Resultado em } \%, 6 \text { do } \\
\text { braco em funçäo do sexa }(\mathrm{N}=118)\end{array}$} \\
\hline Suspersäo & \multicolumn{2}{|c|}{ Feminino } & \multicolumn{2}{|c|}{ Masculivio } & \multicolumn{2}{|c|}{ Total } \\
\hline $\begin{array}{l}\text { Le Braco } \\
\text { (Aec. Seg) }\end{array}$ & Abs. & $\%$ & Abs. & $\%$ & Abs. & $\%$ \\
\hline $2,11-0,6$ & 38 & $64 \%$ & 33 & $56 \%$ & 71 & $60 \%$ \\
\hline $2,89-1,1$ & 17 & $29 \%$ & 14 & $24 \%$ & 31 & $26 \%$ \\
\hline $1,14,1,6$ & 1 & $2 \%$ & 0 & $0 \%$ & 1 & $1 \%$ \\
\hline $1,6: 2,1$ & 1 & $2 \%$ & 6 & $10 \%$ & $?$ & $6 \%$ \\
\hline $2.11 \cdot 2.6$ & 1 & $2 \%$ & 2 & $3 \%$ & 3 & $3 \%$ \\
\hline $2,6,-3,1$ & 0 & $0 \%$ & 0 & $0 \%$ & 0. & $0 \%$ \\
\hline $3,1 / \cdot 3,6$ & 0 & $0 \%$ & 1 & $2 \%$ & 1 & $1 \%$ \\
\hline $3, \leqslant,-4,1$ & 1. & $2 \%$ & 3 & $5 \%$ & 4 & $3 \%$ \\
\hline Tatal & 59 & $100 \%$ & 59 & $100 \%$ & 118 & $100 \%$ \\
\hline
\end{tabular}

entre os 195 e 295 segundos, correspondendo a $87 \%$. Quanto às meninas, o mesmo se sucedeu em relação ao tempo despendido, representando $89 \%$ do grupamento. Em relação as médias entre os sexos, percebe-se que tal qual se vê na FIGURA 8, que o grupo masculino apresentou uma média em torno de 232,50 segundos (s), enquanto o feminino de Percebe-se que a 252,64 segundos. Nesta sociedade prova, quanto maior o estabelece padrões percentual, menor é o de comportamento
rendimento. Pelos dados apresentados, verifica-se diferenciados, que que os meninoscontribuem para apresentaram resultados a desigualdade percentualmente melhores entre os sexos do que as meninas.

Revendo a literatura, CARDOSO (1994), aponta para uma explicação dos laços teóricos que poderiam ter influenciado nos desempenhos explicando que na prática das atividades esportivas e culturais, as atividades mais comportadas, do ponto de vista motor, em que correr, saltar, trepar, cair etc, são atributos permitidos, apenas aos meninos, ficam prejudicadas as meninas por não poderem desenvolver mais seu potencial motor. Com isso, ficam os indivíduos do sexo feminino sem oportunidade de terem as mesmas condições que os do masculino para desenvolver suas habilidades

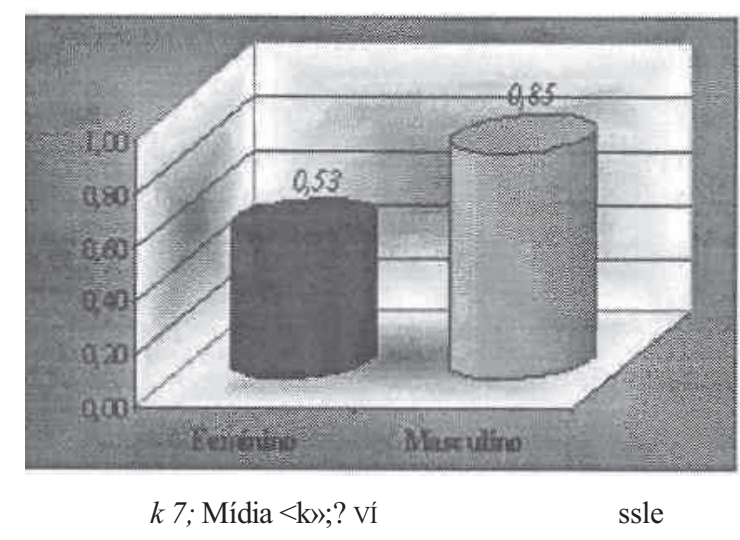




\begin{tabular}{|c|c|c|c|c|c|c|}
\hline \multicolumn{7}{|c|}{$\begin{array}{l}\text { TABELA 8: Resultad o em } \\
\text { funçäo do se xo (N=118) }\end{array}$} \\
\hline Shutile & \multicolumn{2}{|c|}{ Fentinino } & \multicolumn{2}{|c|}{ Masculino } & \multicolumn{2}{|c|}{ Total } \\
\hline $\begin{array}{c}\text { Rum } \\
\text { (dec.Seg) }\end{array}$ & Abs. & 96 & Abs. & 96 & Abs. & 96 \\
\hline $145 \mid-170$ & 0 & $0 \%$ & 2 & $3 \%$ & 2 & $2 \%$ \\
\hline $170 \mid-195$ & 0 & $0 \%$ & 3 & $5 \%$ & 3 & $3 \%$ \\
\hline $1931-220$ & 8 & $14 \%$ & 13 & $22 \%$ & 21 & $18 \%$ \\
\hline $220 \mid-245$ & 18 & $31 \%$ & 24 & $41 \%$ & 42 & $36 \%$ \\
\hline $245 \mid-270$ & 17 & $29 \%$ & 8 & $14 \%$ & 25 & $21 \%$ \\
\hline $270 \mid-295$ & 9 & $15 \%$ & 6 & $10 \%$ & 15 & $13 \%$ \\
\hline $295 \mid-320$ & 4 & 79 & 2 & $3 \%$ & 6 & $5 \%$ \\
\hline $320 \cdot-345$ & 3 & $5 \%$ & 1 & 298 & 4 & 398 \\
\hline Total & 59 & 10098 & 59 & $100 \%$ & 118 & $100 \%$ \\
\hline
\end{tabular}

\begin{tabular}{|c|c|c|c|c|c|c|}
\hline \multicolumn{7}{|c|}{$\begin{array}{l}\text { TABEL A 9: Resultado } \\
\text { funç̃o do sexa }(\mathrm{N}=118)\end{array}$} \\
\hline Cooper & \multicolumn{2}{|c|}{ Femintino } & \multicolumn{2}{|c|}{ Masculino } & \multicolumn{2}{|c|}{ Total } \\
\hline Vo2max & Abs. & $\%$ & Abs. & $\%$ & Abs. & 90 \\
\hline $13,51-19$ & 4 & $7 \%$ & 2 & $3 \%$ & 6 & $5 \%$ \\
\hline $19 \cdot-24,5$ & 16 & $27 \%$ & 7 & $12 \%$ & 23 & $19 \%$ \\
\hline $24.51-30$ & 15 & $25 \%$ & 9 & $15 \%$ & 24 & $20 \%$ \\
\hline $30 \mid-35,3$ & 18 & $31 \%$ & 23 & $39 \%$ & 41 & $35 \%$ \\
\hline $35,51.41$ & 5 & $8 \%$ & 11 & $19 \%$ & 16 & $14 \%$ \\
\hline $411-46,5$ & 1 & $2 \%$ & 2. & $3 \%$ & 3 & $3 \%$ \\
\hline $46,51-52$ & 0 & $0 \%$ & 4 & $7 \%$ & 4 & $3 \%$ \\
\hline $521-57,5$ & 0 & $0 \%$ & 1 & $2 \%$ & 1 & $1 \%$ \\
\hline Total & 59 & $100 \%$ & 59 & $100 \%$ & 118 & $100 \%$ \\
\hline
\end{tabular}

motoras durante as aulas de Educação Física. Os resultados encontrados na amostra estudada permitem aceitar esta premissa.

Dando continuidade à apresentação dos resultados, o último teste analisado foi o teste de Cooper. Para visualizar a comparação entre os sexos construi-se a TABELA 9, que por sua vez denota que neste teste os meninos apresentaram os melhores resultados em relação às meninas. $\mathrm{O}$ sexo masculino teve concentração em forma de 19 a 41 da capacidade de vo ${ }^{2} \max$, ao passo que o feminino exibiu concentração em torno de 19 a 35,5 da capacidade de vo ${ }^{2} \max$.

Pode-se observar, na FIGURA 9, que as médias das meninas se apresentaram em torno de 28,40 e para os meninos, 32,87. Para LOPES (1986, p. 159), uma possível explicação para esse resultado é baseada em dados/questões orgânicas. $\mathrm{O}$

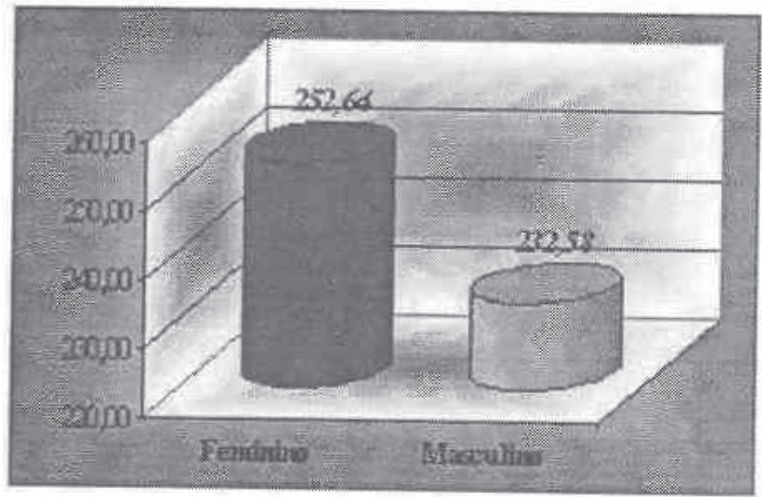

FIGURA 8: Média dos valores obtidos no teste Shuttle Run em funçäo do sex 0 .

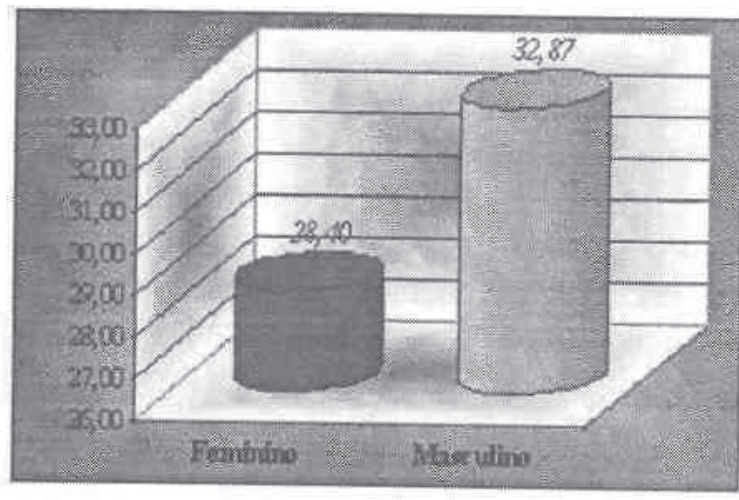

FIGURA 9: Média dos valores obtidos no teste de Cooper em função do sex 0 .

autor enfatiza que "a existencia de hemoglobina em menos quantidade na mulher traz consigo uma conseqüência de grande importância, que está ligada à menor capacidade de transportar oxigênio, já que a hemoglobina é responsável pelo seu transporte e daí uma diminuição da capacidade de prolongar um esforço em resistência aeróbica".

\section{Conclusão}

Os resultados encontrados no estudo conduzem à conclusão de que houve desempenho físico e motor diferenciado entre meninos e meninas diante do Eurofit, o que confirma a literatura revisada. Ficou evidenciado que em oito das nove provas aplicadas, os meninos obtiveram respostas motoras melhores que as meninas, nas provas 
de: a) equilíbrio; b) tocando os discos; c) salto em extensão; d) apreensão manual; e) sente e levante; f) suspensão na barra; g) Shuttle Run; h) resistencia aeróbica. As meninas foram melhores do que os meninos apenas na prova sente e alcance. Na prova de agilidade, tocando os discos, os resultados percentuais apontaram uma pequena diferença em favor do sexo masculino.

Constatou-se que os resultados encontrados dão fortes indícios de que a prática de atividades físicas na escola não tem contribuído para o melhor desempenho das meninas.

Percebe-se que a sociedade estabelece padrões de comportamento diferenciados, que contribuem para a desigualdade entre os sexos. Neste contexto, os fatores culturais podem ter agido como agente influenciadores do desempenho físico e motor de meninos e meninas.

\section{Referências Bibliográficas}

AZEVEDO, Tânia. A mulher na educação física e no esporte. In ROMERO, Elaine (Org). Mulheres em movimento. Vitória: EDUFES, 1997, p.113-135.

BARBANTI, Valdir. Treinamento físico: base científica. 2.ed. São Paulo: CLR Balieiro, 1995.

Treinamento físico: bases científicas. 3.ed. São Paulo: CLR Balieiro, 1996.

BARROS, Daisy. Educação física na escola primária. 4.ed. Rio de Janeiro: José Olímpio, 1986.

BELLOTI, Elena Gianini. Educar para a submissão: o descondicionamento da mulher. 2.ed. Petrópolis: Vozes, 1985-

BIAGGIO, Angela M. Brasil. Psicologia do desenvolvimento. Petrópolis: Vozes, 1985.

BOHME, Maria Tereza. Zur entwicklung der kõrperlichen fítness brasiliamscher kinder und jugendlicher. Colônia, 1993. Tes
(Doutorado), Universidade Justus-Liebig Gressen.

. Aptidão física: importância e relações

com

a educação física. Viçosa, v.2, n. ${ }^{\circ}$ 10, p. 17-25, 1994.

BRANDÃO, Maria Regina e FIGUEIRA JÚNIOR, Ayíton. Performance esportiva: uma análise multidimencional. Revista Treinamento Desportivo, São Paulo, v.1, n. ${ }^{\circ}$ 1, p.58-72, 1996.

BRUHNS, Heloisa T Corpos femininos na relação com a cultura. In ROMERO, Elaine (Org). Corpo, mulher e sociedade. Campinas: Papirus, 1995,p.71-98.

CARDOSO, Fernando L. O gênero e o movimento humano. Revista Brasileira de Ciências do Esporte. Santa Maria, RS., v.15, n. ${ }^{\circ} 3$, p.265268, jan., 1994.

COOPER, Kenneth, H. O programa aeróbico para o bem estar total. 3.ed. Rio de Janeiro: Nórdica, 1982.

. O programa aeróbico para o bem estar total. 3.ed. Rio de Janeiro: Nórdica, 1986.

CORBIN, C. B. Youth fitness, exercise and health: there is much to be done. Research Quarterly for Exercise and Sports, v. 58, n 4, p.315-20, 1987.

CUNHA, Manuel Sérgio. Para uma epistemologia da motricidade humana. 2.ed. Lisboa: Compedium, 1994.

DANTAS, Estélio. Fitness. A ecologia do corpo. Projeto de Pesquisa. Programa de PósGraduação. Rio de Janeiro: UCB, 1987 (mimeo).

DAOLIO, Jocimar. A construção cultural do corpo feminino ou o risco de transformar meninas em antas. In ROMERO, Elaine (Org). Corpo, mulher e sociedade. Campinas: Papirus, 1995, p.99-108.

GUEDES. Dartagnan e BARBANTI, Valdir. 
Desempenho motor em crianças e adolescentes.

Revista Paulista de Educação Física. São Paulo, v.9, n. ${ }^{\circ} 1$, p.37-50, jan./jun., 1995 -

KNAPP, Barbara. Desporto e motricidade. Lisboa: Compedium, s/d.

LOPES, Pinto. A mulher e o desporto. Horizonte Revista de Educação Física e Desporto. Lisboa, v. II, n. ${ }^{\circ} 11$, p. 157-160, Jan/Fev, 1986.

MYOTIN, Emmi. A participação da adolescente brasileira em esporte e atividades físicas como forma de lazer: fatores psicológicos e socioculturais. In ROMERO, Elaine (Org). Corpo, mulher e sociedade. Campinas: Papirus, 1995, p. 177-195.

PELLEGRINI, Ana M.; GREENDORFER, Suzan L.; e BLINDE, Elaine M. Fatores determinantes do envolvimento da criança em atividades físicas e esportivas. Cadernos de Pesquisa. São Paulo, n. ${ }^{\circ} 60$, p.38-44, fev., 1987.

PIKUNAS, J. Desenvolvimento humano. 3 ed. São Paulo: MC GRAW HILL do Brasil, 1987.

POLLOCK,L. M e WILMORE.H.J. Exercícios na saúde e na doença: avaliação e prescrição para prevenção e reabilitação. $2^{\mathrm{a}}$ ed. Rio de Janeiro: Editora Médica e Científica Ltda., 1993.

POMPEU, Fernando A. Considerações biológicas sobre o treinamento de crianças e adolescentes. Revista Mineira de Educação Física. Viçosa, v.3, n. ${ }^{\circ}$ 1,p.43-64, 1995.

ROMERO, Elaine. A educação física a serviço da ideologia sexista. Revista Brasileira de Ciências do Esporte, v. 15, n. 3, Jun, p.226-234, 1994. . A arquitetura do corpo feminino e a produção do conhecimento. Corpo, mulher e sociedade. Campinas: Papirus, 1995, p.235270.

SCOTT, Joan. Gênero: uma categoria útil de análise histórica. Revista Educação \& Realidade. Porto Alegre, v.16, n. ${ }^{\circ}$ 2, p.5-22, jul/dez, 1990.

WEBER, Gerlinde. A questão de gênero. Pró-
Discente. Programa de Pós-graduação em Educação. Vitória: UFES, v.3, n. ${ }^{\circ}$ 6, p.39-45, abr., 1997.

\section{Notas}

${ }^{1}$ Professora do Colégio de Aplicação Dr Paulo Gissoni e Colégio Castelo Branco da Universidade Castelo Branco -RJ

${ }^{2}$ Professora Titular do Programa de Pós-Graduação em Ciências da Motricidade Humana, Universidade Castelo Branco- RJ.

Avenida Santa Cruz, 1631 - Realengo - RJ.

CEP. 21710-250. 